\title{
La función administrativa en la era de las TIC*
}

\author{
Josías Daniel Mojica Domínguez ${ }^{* *}$, Adalberto Barandica Domínguez ${ }^{* * *}$, Lena Rodero Acosta****, \\ Mónica Franco Montenegro ${ }^{\star * \star \star}$, Hugo Hernández Palma ${ }^{\star \star \star \star * \star}$, Sebastián Arboleda Cardona ${ }^{\star \star \star \star * \star *}$
}

\section{Resumen}

Una sociedad globalizada como la de hoy plantea, a lo menos, dos retos al campo del derecho: uno está determinado por la crisis de fundar una estructura jurídica bajo la noción del derecho de los Estado-nación; y el otro reto está relacionado con la cultura del reconocimiento y respeto de los derechos humanos. A partir de esta problemática, la investigación tiene como objetivo analizar la realidad de la función administrativa como una herramienta de trasformación de la democracia en contextos de pertinencia y eficiencia. Este artículo es el resultado de una investigación de metodología cualitativa, investigación documental, a través de un proceso crítico de revisión de archivos (información numérica y no numérica) y análisis de contenidos, apoyado en la triangulación de prácticas profesionales, estructuras funcionales del derecho y la implementación de normas sustantivas. Es necesario analizar los niveles de participación ciudadana a través de la integración de los procesos sociales y judiciales, así como de las posibilidades de acceso a la información, generando una proyección de los niveles de transparencia y respeto a la garantía fundamental del debido proceso.

Palabras clave: globalización, redes sociales, democratización, transnacionalización, constitucionalización.

\section{Administrative function in the era of ICTs}

\begin{abstract}
A globalized society, like the one of today, proposes at least two challenges to the field of law. One is determined by the crisis of creating a juridical structure under de State-Nation notion and the other challenge is related to the culture of recognition and respect for human rights. From this problem, this research work aims to analyze the reality of administrative function as a tool for the transformation of democracy in contexts of pertinence and efficiency. This article is the result of a qualitative research work -a documentary research- through a critical process of revision of archives (numeric and non-numeric) and a content analysis, supported by the triangulation of professional practices, functional structures of the law and the implementation of substantive rules. It is necessary to analyze the levels of participation of the citizens through the integration of the social and judiciary processes, and of the possibilities to access to the information, generating a projection of the levels of transparency and respect to the basic guarantee of the due process.
\end{abstract}

key words: globalization, Social networks, democratization, transnationalization, constitutionalising.

\footnotetext{
* Artículo de reflexión resultado de trabajo cooperado entre el Grupo de Investigación Perspectiva Constitucional de la Función Administrativa de la Universidad del Atlántico, Cienticol COL0162272 y Derecho, Justicia y Estado Social de Derecho, de la Corporación Universitaria Americana COL0088246

** Abogado, Docente investigador del Universidad del Atlántico, Magister en Derecho de la Universidad Nacional de Colombia. Contacto: josiasmojicadominguez@gmail.com

*** Abogado, Docente TC investigador del Universidad del Atlántico, Magister en Derecho de la Universidad Nacional de Colombia. Mail: barandicaadalberto@hotmail.com

**** Abogado, Docente Ad honoren y Decana del Programa de Derecho de la Universidad del Atlántico. email. Contacto: lenarodero@mail.uniatlantico.edu.co

**** Psicologa, Universidad del Norte. Magister en Educación (Cognición) Universidad del Norte. Docente investigadora Corporación Universitaria Universidad de la Costa. Grupo de investigación Cultura, Educación y Sociedad. mail: mfranco@cuc.edu.co.

****** Ingeniero Industrial, Magíster en Sistemas de Gestión, Especialista en estudios Pedagógicos, docente investigador Grupo Gemas Universidad Simón Bolívar. hugoghernandezpalma@gmail.com

****** Abogado de la Universidad de Medellín (2012). Especialista en Derecho Comercial Universidad del Rosario (2014), Candidato a Magíster en Propiedad Intelectual y Derecho de las Nuevas Tecnologías (2015), Docente Director del Consultorio Jurídico Lasallista del Programa de Derecho de la Facultad de Ciencias Sociales y Educación de la Corporación Universitaria Lasallista. Docente investigador, miembro del grupo de investigación en derecho GRIDE de la Corporación Universitaria Lasallista.
}

Autor para correspondencia: Josías Daniel Mojica Domínguez, email: josiasmojicadominguez@gmail.com Artículo recibido: 27/07/2015; Artículo aprobado: 18/08/2015. 


\section{A função administrativa na era das TIC}

\section{Resumo}

Uma sociedade globalizada como a de hoje, planta ao menos, dois reptos ao campo do direito, um está determinado pela crise de fundar uma estrutura jurídica sob a noção do direito dos Estado-Nação; e o outro objetivo, está relacionado com a cultura do reconhecimento e respeito dos direitos humanos. A partir desta problemática, a investigação tem como objetivo analisar a realidade da função administrativa como uma ferramenta de transformação da democracia em contextos de pertinência e eficiência. Este artigo é o resultado de uma investigação de metodologia qualitativa, investigação documentário, através de um processo critico de revisão de arquivos (informação numérica e não numérica) e análise de conteúdos, apoiado na triangulação de práticas profissionais, estruturas funcionais do direito e a implementação de normas substantivas. É necessário analisá-los níveis de participação cidadã através da integração dos processos sociais e judiciais, assim das possibilidades de acesso à informação, gerando uma projeção dos níveis de transparência e respeito à garantia fundamental do devido processo.

Palavras chaves: globalização, Redes Sociais, Democratização, Transnacionalização, constitucionalização.

\section{Introducción}

Los avances tecnológicos que en materia de telecomunicaciones (Internet, televisión, celular) ha tenido la humanidad han acelerado el proceso de globalización que, por aproximarnos a una fecha, desde mediados del siglo pasado avanza a pasos gigantescos. Bajo esa dinámica, hoy en día, el concepto de distancia se ha relativizado, en razón a la vertiginosa resignificación de este concepto y sus implicaciones en el desarrollo sostenible y sustentable de los países; además, la implementación de las nuevas tecnologías de las comunicaciones han determinado un cambio drástico en las relaciones humanas entre las distintas sociedades, ya que, mientras por una parte se acortan las distancias entre sujetos o sociedades, por el otro lado, se despersonalizan las relaciones humanas.

Así las cosas, la cultura del respeto y la conciencia, desde la Administración Pública, implica la dinamización del derecho, articulado con un tipo de funcionario que permita la eficiencia y la eficacia de la función administrativa, no solo desde la participación de las personas, sino desde el acceso a la información de manera oportuna, para la solución alternativa de conflictos y la economía en la gestión de las políticas del Estado. De esta manera, se podrán plantear soluciones integrales de gestión pública, capaces de dar respuesta a las expectativas y exigencias de los mercados y de la sociedad.

\section{Metodología}

El presente trabajo se enmarca dentro de la metodología cualitativa, investigación documental, según la cual, la revisión de archivos (información numérica y no numérica) y el análisis de contenido se convierten en técnicas fundamentales para comprender el significado de las situaciones de tensión alrededor del objeto de estudio frente a la vida cotidiana.

En el primer escenario se diseñó la investigación (definición del tema, delimitación conceptual, temporal y espacial), articulando una revisión de estudios y de literatura relacionada, que permitiera establecer qué se ha dicho sobre el tema propuesto, desde qué punto de vista y con qué resultados. Consecuentemente, se sistematizaron los resultados de dicha búsqueda, gestionando los saberes y las dinámicas jurídicas de las diferentes instancias.

Asimismo, se desarrolló un proceso de triangulación entre las prácticas judiciales y la implementación de normas sustantivas y se realizó la búsqueda y selección de información, lo cual exigió el rastreo e inventario de los documentos existentes y disponibles y de las fuentes complementarias, en bibliotecas y centros de documentación. Por lo tanto, para adelantar esta investigación se concentró la mirada en la situación problema planteada y en sus contextos particulares, sus singularidades y sus tipologías. 


\section{Desarrollo}

Para ilustrar la problemática en esta investigación es necesario partir de una aproximación, si se quiere, a priori y sin exhaustividad, al fenómeno de la globalización, concepto complejo del cual no se puede tener una definición unívoca, debido a que encierra una serie de categorías sociales y, a la vez, se convierte en un punto de encuentro de distintas disciplinas del conocimiento como la economía, la sociología, las ciencias ambientales, y el derecho, por cuanto es necesario un escenario de comprensión del proceso social y sus niveles de afectación, promoviendo la motivación, directa o indirecta, del cambio el curso del desarrollo económico y social como vehículo de generación de nuevas formas de vida en las sociedades.

A manera de ejemplo, se ha manifestado la globalización desde el modelo económico imperante en el mundo que es el de libre mercado o "neoliberalismo". Este modelo ha propiciado que empresas muy poderosas de países desarrollados se hayan posicionado en todos los continentes, y que en su dinámica de mercadeo se hayan adaptado a las culturas locales, para generar en los consumidores locales empoderamiento y cariño por la empresa; tales son los casos de Coca-Cola ${ }^{\circledR}$, y McDonald ${ }^{\circledR}$. Esa dinámica y transformación de esas empresas se ha denominado "transnacionalización" o empresas transnacionales que responden al nuevo concepto económico de "mercado global", entendiéndolo como un escenario de actores económicos transnacionales en donde caben empresas, instituciones y redes, además de organismos como la OMC, los tribunales de arbitramento internacionales, la Lex Mercatoria, que propician la generación de un nuevo derecho, que se configura y nace en el mercado global, y que incide y determina las relaciones jurídicas locales (como relaciones jurídicas locales aquellas que se dan dentro de un Estado-nación).

La globalización en el derecho pone en crisis categorías jurídicas como la soberanía en los llamados Estados-nación, los cuales pueden ser vistos desde dos campos en los que se tocan las bases teóricas sobre las cuales se ha fundamentado el concepto de soberanía:
Un primer campo está determinado por el mercado global. En ese juego de actores transnacionales del mercado, surge la necesidad de control, ya que un mercado sin controles puede generar un estado de crisis económica, tal como sucedió en 1929 y en 2007.

Hoy día, el mercado global busca generar reglas de control mediante organismos como la Organización Mundial del Comercio y los tribunales internacionales de arbitramento; asimismo, los Estados-nación han propiciado la integración de los mercados, caso de Europa con la creación del mercado común (Unión Europea); también están experiencias significativas de otras comunidades desde América Latina, dentro de las que se encuentran el Mercosur y la Comunidad Andina de Naciones (CAN).

Estas integraciones económicas han tratado de ser escenarios de control del mercado global o transnacional; sin embargo, las dinámicas del mercado global ponen de manifiesto las limitaciones de los Estado-nación de controlar los actores trasnacionales del mercado (De la Reza, 2006, 68).

Un segundo campo está determinado por el reconocimiento y respeto de los derechos humanos. Desde mediados del siglo XX con la Declaración Universal de los Derechos Humanos, se dio paso a una ola de declaraciones, pactos y tratados sobre derechos humanos, que los convirtieron en un bloque de freno y control en contrapeso al ejercicio arbitrario del poder en los Estadonación; adicionalmente, estas declaraciones de derechos humanos sirvieron de fundamento para la creación de sistemas internacionales de justicia, como el sistema interamericano de derechos humanos, integrado por la Comisión Interamericana de Derechos Humanos y la Corte Interamericana de Derechos Humanos, y en el ámbito mundial se destaca la Corte Penal Internacional.

Este segundo campo tiene incidencia directa sobre dos disciplinas del derecho. Por un lado, el derecho constitucional, cuyas estructuras dogmáticas han sido tocadas por la teoría del neoconstitucionalismo, pero para el propósito 
de esta investigación se asume como un constitucionalismo marcado por el respeto de las garantías de los derechos humanos por parte del Estado en sus distintas funciones, lo cual implica que la Constitución adquiere un valor normativo que irradia las distintas ramas del derecho (administrativo, privado, penal, etc.) y a ella están sometidas todas las autoridades estatales (Bernal, C. (2009). El neoconstitucionalismo y la normatividad del derecho. Bogotá: Universidad Externado de Colombia).

Para ilustrar esa transformación en las ramas del derecho, se pone como ejemplo el derecho administrativo, el cual ha sido afectado en su dogmática, en dos sentidos: de un lado, por la noción del neoconstitucionalismo, lo que implica que la función administrativa del Estado debe ejercerse bajo el respeto de las garantías y principios fundamentales de los derechos humanos y de la democracia. Ello implica que todas las actuaciones estatales deben ser guiadas por el principio del debido proceso y la transparencia, y por la democratización ${ }^{1}$, y de otro lado, que las construcciones dogmáticas de instituciones jurídicas propias, como la teoría del acto administrativo, deben ajustarse al nuevo ideario neoconstitucional ${ }^{2}$.

La democratización ${ }^{3}$, como categoría teórica asumida bajo este análisis, implica dos nociones: la primera, relacionada con los niveles de participación ciudadana o de los administrados en las decisiones o actuaciones de las autoridades administrativas del Estado. En ese sentido, ponemos como ejemplo, las consultas ciudadanas, las veedurías ciudadanas, etc.; la otra noción va relacionada con los niveles de legitimidad que tenga el ejercicio de la función administrativa por parte de los ciudadanos. Es decir, que si bien es cierto que la función administrativa goza de la presunción de legalidad, hasta tanto no sea debatida en proceso judicial, esa función administrativa debe gozar de legitimidad en sus administrados, lo cual genera que las actuaciones estatales serán eficaces para los propósitos por los cuales fueron expedidas.

Esa doble vía de la democratización de la función administrativa del Estado puede ser visionada desde dos puntos de vista. El primero, desde la ciudadanía, al analizar cómo la participación de la ciudadanía en los procesos administrativos y el acceso a la información generan niveles de transparencia $y$, por ende, respeto a la garantía fundamental del debido proceso. El segundo, desde la administración, identificando que una de las preocupaciones de la Administración Pública bajo el sometimiento total a las garantías fundamentes de los derechos humanos es poder lograr niveles de legitimación de las medidas tomadas.

Ambas miradas, desde el plano teórico, nos llevan a un solo punto en el plano práctico de una sociedad moderna, determinada por la globalización, por las redes sociales como mecanismos de expresión, y es plantearnos el siguiente problema: ¿Cómo en una sociedad de las comunicaciones y las redes sociales, se logra la democratización de la función administrativa?

A manera de hipótesis sobre el anterior interrogante, se considera que utilizando las lógicas de las necesidades, una sociedad puede que no sea tan consciente de la necesidad de democratización y participación en lo público, cuando sus prioridades están determinadas por la satisfacción de las necesidades básicas del conglomerado y de todas aquellas que generen un estado de bienestar; en ese entendido, su interés en lo público está determinado por los efectos adversos de medidas administrativas sobre sus condiciones de vida.

1 Manuel Atienza en un ensayo denominado Constitucionalismo, globalización y derecho pone en evidencia la problemática en el campo del constitucionalismo, y cómo las tendencias actuales de esta disciplina podrían resultar consecuencias del proceso globalizador. En CARBONELL, Miguel y García Jaramillo, Leonardo. El canon neoconstitucional. Madrid, Trota, 2010.

2 Para una mayor comprensión de la constitucionalización de la teoría del acto administrativo, ver MOJICA DOMíNGUEZ, Josías Daniel (2013). El acto administrativo: Discurso constitucional en el Estado social de derecho. Bogotá, Colombia. Universidad Nacional de Colombia. Editorial Ibáñez,

3 El término puede verse una noción de Habermas, Nonet y Selznick, las cuales señala Gunter Teubner en el texto La fuerza del derecho, p. 127, Bogotá, Siglo del Hombre, 2000, para tratar de explicar cómo en un mundo postmoderno, el capitalismo causa unas crisis sistémicas y que en el derecho se representa en crisis de legitimidad. 
De tal suerte, una salida a esa manifestación de poco interés puede ser la implementación del principio de responsabilidad virtual. Este escenario podría ser entendido como, "soy responsable de los efectos negativos de las medidas administrativas en tanto no participe en el discurso público virtual". Implementar un principio como este conduce a generar en el uso de las redes y tecnologías de comunicación una cultura cívica, es decir, propicia la formación de un ciudadano virtual.

Para el desarrollo de la investigación, de la cual se desprende este ensayo, y utilizando una metodología cualitativa, se mostrará, en primer lugar, cómo la globalización a través de los avances de las nuevas tecnologías de la información y la comunicación ha incidido en la forma de interrelación de la sociedad de hoy; en segundo lugar, mostraremos cómo las nuevas dinámicas de una sociedad marcada por las TIC generan un replanteamiento de la democracia; y por último, pretendemos que la Administración Pública puede asumir los desafíos que plantea una sociedad determinada por las TIC y la globalización.

Las nuevas tecnologías de la información: una herramienta de la globalización para la transformación de la sociedad de hoy

La globalización es un concepto muy difícil de definir unívocamente, pero se muestra como un fenómeno que trasciende todas las esferas de la vida social del hombre. Por ello, para efectos prácticos de esta investigación, se hace referencia a la definición de Giddens (2004) quien trata de mostrarla como una dinámica que elimina y va diluyendo la importancia de las distancias y las divisiones territoriales, dejando como consecuencia una nueva organización del tiempo, la distancia y el espacio en las relaciones a escala global.

Este fenómeno se ha de analizar como categoría determinante del actual auge de las redes sociales, ya que según Ulrich Beck (2008) es un proceso complejo, cuyo estudio no puede hacerse aislado desde las distintas manifestaciones de la globalidad, sino que debe hacerse interdependientemente. Para justificar esto, señala ocho razones. Textualmente indicó:
Un diferenciador esencial entre la primera y la segunda modernidad es la irrevisabilidad de la globalidad resultante. Lo cual quiere decir lo siguiente: existe una afinidad entre las distintas lógicas de las globalizaciones ecológica, cultural, económica, política y social, que no son reductubles -ni explicables- las unas a las otras, sino que, antes bien, deben resolverse y entenderse a la vez en sí mismas y en mutua interdependencia. (...) Pero ¿Qué es lo que hace irrevisable la globalidad? He aquí ocho razones, introducidas con frases programáticas:

1. El ensanchamiento del campo geográfico y la creciente densidad del intercambio internacional, así como el carácter global de la red de mercados financieros y del poder cada vez mayor de las multinacionales;

2. La revolución permanente en el terreno de la información y las tecnologías de la comunicación;

3. La exigencia, universalmente aceptada, de respetar los derechos humanostambién considerada (de boquilla) como el principio de la democracia;

4. Las corrientes icónicas de las industrias globales de la cultura;

5. La política mundial posinternacional y policéntrica: junto a los gobiernos hay cada vez más actores transnacionales con cada vez mayor poder (multinacionales, organizaciones no gubernamentales, Naciones Unidas);

6. El problema de la pobreza global;

7. El problema de los daños y atentados ecológicos globales;

8. El problema de los conflictos transculturales en un lugar concreto (BECK, Ulrich. 1997, 34-36).

Tales aseveraciones, demuestran como esa complejidad en la globalización digital, en el campo de las tecnologías de la información y comunicación (en adelante TIC), permite el paso de las redes y modos de vida de una sociedad hacia un sistema dependiente 
y conflictivo, toda vez que no solo afecta el plano económico, sino también su forma de interacción, imposibilitando que el ejercicio democrático sea libre y espontaneo.

En este campo de las TIC, desde finales de los años noventa, el uso de Internet se ha multiplicado de manera exponencial, y esto en gran medida ha sido producto de la creación de aplicativos de comunicación instantánea como lo son las redes sociales. Según estadísticas tomadas de la página oficial de Facebook en el mundo hay 1440 millones de usuarios activos mensuales y unos 936 millones de usuarios activos diarios, y adicionalmente 1250 millones de usuarios se conectan a través de dispositivos móviles ${ }^{4}$. En el caso de Twitter, esa red social registra 302 millones de usuarios activos mensuales, y un tráfico de 500 millones de tweets al día, cifras reportadas a 31 de marzo de 2015, por la página oficial de esa compañía ${ }^{5}$.

Para el año 2012, según la encuesta realizada por el Ministerio de las TIC y la firma Ipsos Napoleón Franco, sobre el consumo digital, se pudo establecer que 8 de cada 10 colombianos usa Internet, que el $54 \%$ de los colombianos se conecta a Internet todos los días; adicionalmente, que el $64 \%$ de los hogares de las ciudades con población de más de 200 mil habitantes cuenta con conexión a Internet; en igual sentido, en los estratos socio-económicos 1 y 2 se ha mostrado un aumento en el acceso a Internet en un $17 \%$ respecto al año 2010. Respecto al uso de las redes sociales, esa encuesta también indicó que 6 de cada 10 colombianos visitaban redes sociales, y de ese porcentaje, el $98 \%$ tiene cuenta en Facebook y el $31 \%$ tiene cuenta en Twitter (IPSOS Napoleón Franco, 2012).

Posteriormente, un estudio ${ }^{6}$ de la Comisión de Regulación de las Telecomunicaciones muestra el siguiente comportamiento de la sociedad colombiana frente al uso del Internet: para el año 2010 , el $40.8 \%$ de los colombianos accedían todos los días a internet, el $47 \%$ lo hacia una vez a la semana y, el $9.8 \%$ una vez al mes. Para el año 2011 el $41.9 \%$ accedía a internet todos los días, el $47.1 \%$ una vez por semana y, el $9.2 \%$ una vez al mes. Para el año 2012 el $46 \%$ accedía todos los días a internet, el $42.6 \%$ una vez por semana y, el $11.3 \%$ una vez al mes. Para el año 2013, 48.3 \% accedía todos los días a internet, el $41.6 \%$ lo hacia una vez a la semana y, el $8.8 \%$ una vez al mes.

Asimismo, la infraestructura y acceso a las TIC evidencia que en materia de conectividad mediante telefonía móvil celular, hay 106.7 suscriptores por cada 100 habitantes; Internet banda ancha (conexiones fijas) ha mostrado un aumento de $5.9 \%$ en el 2010 y de $9.5 \%$ en el 2013.

El estudio también indica que el $42.2 \%$ de los hogares colombianos tiene computador de escritorio, portátil o tableta, lo que ha significado un aumento en el acceso a Internet de $35.7 \%$ en el 2013 frente a un $19.3 \%$ en el 2010. Otro dato interesante de ese estudio es que en materia educativa la relación de computadores por estudiantes bajó, es decir, mientras que en el año 2010 había 20 estudiantes por computador, al año 2013 solo había 11 estudiantes por computador, lo que representó un descenso del $45 \%$. Esto sin duda representa un avance en el acceso a las nuevas tecnologías de la información; sin embargo, la relación sigue siendo alta, ya que solo con una cobertura del $100 \%$ de acceso a las TIC'S se puede afirmar que existe el espacio necesario para una democratización del ejercicio de la función administrativa, lo cual en principio garantiza la oportunidad de participar por parte de todos los ciudadanos en las decisiones de su interés.

Por otra parte, esta paulatina digitalización de la sociedad colombiana también se evidencia en la implementación de escenarios virtuales de interacción del Estado con sus personas, por cuanto el Estado colombiano implementó la estrategia de Gobierno en Línea (GEL), la cual, según el estudio citado, muestra

\footnotetext{
//newsroom.fb.com/company-info/.

//about.twitter.com/company

Comisión de Regulación de las Telecomunicaciones. Avance de Colombia en la sociedad de la información. Julio 2014.
} 
que al año 2013 el $100 \%$ de las entidades gubernamentales tienen presencia en Internet con su propio sitio web o en el sitio web de otra entidad estatal. Igualmente, el $100 \%$ de las entidades gubernamentales ofrece plataforma de servicios al usuario, y el $100 \%$ de dichas entidades ofrece servicios en línea.

Ahora bien, estas estadísticas, relacionadas con las cifras internacionales sobre acceso a Internet y a las telecomunicaciones, muestran a Colombia en la siguiente situación: según cifras de internetworldstats.com (referenciadas en informe del Ministerio de las Tic) en el mundo hay 2400 millones de usuarios de Internet. De esos, en una relación habitantes por país, Colombia muestra que el $49 \%$ de sus habitantes tiene acceso a Internet, mientras los países miembros de la OECD, tiene un $73 \%$ de sus habitantes con acceso a Internet; es visible también, que la región de Latinoamérica y el Caribe muestra un $44 \%$, y la media en el mundo está en un $36 \%$ de los habitantes por país ${ }^{7}$. No obstante, respecto a las redes sociales en el mundo, las cifras registran que Colombia ocupa el lugar número 15 con más usuarios de Facebook, con cerca de 20 millones de usuarios, y ocupa el puesto 14 con más usuarios de Twitter con cerca de 5,2 millones de usuarios.

Todas las anteriores cifras inciden en el ranking del índice de desarrollo tecnológico del Foro Económico Mundial, el cual indica que al año 2013, Colombia se ubicó en el puesto 66 de 144 países, escalando 7 posiciones respecto al año anterior, y estando mejor posicionado que países de la región como Argentina (puesto 99), Perú (puesto 103), Venezuela (puesto 108) y Nicaragua (puesto 125$)^{8}$.

$\mathrm{Al}$ analizar las cifras anteriormente señaladas con los cambios en los patrones de comportamiento de la sociedad de hoy, hay una directa relación entre el avance tecnológico y el comportamiento social de lo instantáneo.

Adicionalmente, esas cifras muestran que el avance y el mayor uso de las TIC propician un mayor ejercicio democrático, de respeto por garantías fundamentales y de ejercicio del poder de manera transparente. Sin embargo, como más adelante se mostrará, esa tendencia presenta una paradoja: mientras muchas más personas en el mundo tienen más rápido y fácil acceso a la información, están interconectadas permanentemente, y la comunicación instantánea hace el mundo más pequeño, la participación ciudadana y la cultura cívica van en descenso, es decir, no se participa en gran medida en las actividades de la Administración Pública y de la gestión de la sociedad y su economía (Hernández, 1988).

\section{La democracia en una sociedad global y tecnológica}

La paradoja anteriormente planteada entre el mayor volumen de virtualización de las personas y el descenso en la participación cívica es la trampa que va inmersa dentro del proceso de la globalización. Para dar cuenta de esta situación, es necesario hacer un breve repaso conceptual sobre la democracia, sus tipologías y las posibilidades y apuestas por una democracia virtual.

La democracia como forma de gobierno por parte del pueblo ha existido desde la antigua Grecia, pasando por Roma, posteriormente con el auge filosófico de la llustración y por último después de la Segunda Guerra Mundial. En cada época histórica, la democracia como forma de gobierno "por el pueblo y para el pueblo" ha sido desafiada por los contextos espaciales, económicos, culturales, etc. Por ello, para ilustrar cómo se dan esos desafíos a esta forma de gobierno, en el contexto social actual, de una sociedad global, es necesario hacer referencia a los cinco interrogantes fundamentales que se ha planteado el profesor Robert Dahl, al analizar la democracia. Estos son:

1. ¿Cuál es la unidad o asociación adecuada en la que debería establecerse un gobierno democrático? ¿Un pueblo? ¿Una ciudad? ¿Un país? ¿Una sociedad comercial? ¿Una universidad? ¿Una organización internacional? ¿Todos ellos?;

7 Datos tomados de databank en http://www.mintic.gov.co/portal/604/w3-article-4425.html

8 Cifras tomadas del The Global Information Technology Report 2013, Foro Económico Mundial, en http://www.mintic.gov.co/portal/604/ w3-article-4425.html. 
2. Dada una asociación adecuada, ¿Quiénes entre sus miembros deberían gozar de ciudadanía plena? En otras palabras, ¿qué personas deberían constituir el demos? ¿Todos los miembros de la asociación tienen derecho a participar en su gobierno? ¿debería el demos incluir a todos los adultos? Si incluye únicamente a un subconjunto de la población adulta, ¿cuán pequeño puede ser el subconjunto para que la asociación no deje de ser una democracia y se torne en algo diferente, como una aristocracia (el gobierno de los mejores, aristos) o una oligarquía (el gobierno de unos pocos, oligos)?

3. Asumiendo que existe una asociación apropiada y un demos adecuado, ¿cómo han de gobernar los ciudadanos? ¿Qué organizaciones o instituciones políticas necesitarán? ¿Diferirán estas instituciones en los diferentes tipos de asociación?

4. ¿Qué opiniones deberían prevalecer, y en qué circunstancias? ¿Debería prevalecer siempre la mayoría o, en ocasiones, las minorías deberían estar facultadas para bloquear o imponerse sobre el gobierno de la mayoría?

5. Si lo común es que prevalezca una mayoría, ¿Qué habrá de constituir una mayoría adecuada? ¿Una mayoría de todos los ciudadanos? Una mayoría adecuada, ¿debería comprender a grupos o asociaciones de ciudadanos, como por ejemplo, grupos hereditarios o asociaciones territoriales, o a ciudadanos individuales? (DAHL, Robert. 2004: 26).

Esos anteriores interrogantes resumen los puntos álgidos de los debates de los teóricos de la ciencia política sobre cuáles modelos de Estado y sistemas de gobierno se ajustan mejor al concepto de democracia, y qué tipo de democracia (directa o representativa) es mejor para los destinos de una nación. Hagamos el ejercicio en una sociedad global como la de hoy.

Frente al primer interrogante: sin duda, una sociedad global como la de hoy plantea severa dificultad para determinar cuál es la unidad - asociación adecuada en la que debería establecerse un gobierno democrático, ya que la dinámica de la transnacionalización de esferas de poder económico, político, cultural y jurídico, pone en evidencia que los límites de las esferas de poder se han diluido. Ya no es fácil determinar fronteras de soberanía; estos ponen de manifiesto la crisis de la noción de cuerpos normativos nacionales e internacionales, es decir, que el concepto de soberanía de Estado-nación está en crisis.

William Twining ha mostrado la anterior situación, indicando que la globalización presenta tres desafíos a la teoría jurídica. Primero: "desafía las que he denominado "teorías de caja negra", es decir, aquellas que tratan a los Estado-nación, las sociedades, los sistemas jurídicos y los ordenamientos jurídicos como si fuesen entidades cerradas, impermeables, que se pueden estudiar aisladamente"; segundo: desafía la idea de acuerdo con la cual el estudio del derecho y de la teoría jurídica se puede restringir a dos tipos de ordenamiento jurídico: el derecho estatal y el derecho internacional público, concebido como aquel que se ocupa de las relaciones entre los Estado-nación; y, por último, desafía la suficiencia y validez del marco y el vocabulario conceptuales del discurso jurídico (el discurso del derecho y el discurso acerca del derecho) para discutir y explicar fenómenos jurídicos que atraviesan las jurisdicciones, las tradiciones y las culturas (Twining, W. 2003, 121). Bajo esa idea de Twining, queda aún más claro que responder al primer interrogante es muy complejo, porque, ¿Cómo determinamos el espacio en el cual debería establecerse la democracia, cuando los niveles de poder e intervención están dados pero son invisibles?

Frentealos interrogantes2y3. ¿Quiénes entresus miembros deberían gozar de ciudadanía plena? ¿Qué organizaciones o instituciones políticas necesitarán? ¿Diferirán estas instituciones en los diferentes tipos de asociación? En una sociedad global, es necesario indicar que la inmensa mayoría participa de la información, como ya lo hemos dicho, a través de las redes sociales y los accesos virtuales y digitales, lo cual, prima facie, nos indica que todas las personas que utilizan el Internet deberían participar de las decisiones públicas. Sin embargo, esa situación plantea una problemática, y es, ¿hasta dónde la trivialización de la información mediante las redes sociales, disminuye la profundidad del debate público? 
Frente al cuarto interrogante. ¿Qué opiniones deberían prevalecer, y en qué circunstancias? Bajo el dominio de las redes sociales en la sociedad de hoy, es complejo determinar si las decisiones de las mayorías, o las opiniones mayoritarias sobre un asunto público, son necesariamente consecuentes con los principios culturales de una nación o región, ya que muchas veces, la moda, o las tendencias son determinadas por grupos minoritarios pero con mayor poder, que alienan en dicha tendencia a las masas de personas que están en la era digital. Otras veces, las mayorías opacan las decisiones u opiniones sensatas de unas minorías. Por ello, el punto de ponderación entre la una y la otra resulta problemático, dado que lo que realmente determina las decisiones son los niveles de poder.

Frente al quinto y último interrogante. ¿Qué habrá de constituir una mayoría adecuada? Sobre este particular, el efecto que causan las redes sociales en la democracia es que brindan el espacio para que se puedan dar o tomar decisiones a partir de la participación de todos los intervinientes, es decir, las mayorías; y esa participación podría generar una democratización en doble vía: una, desde el poder público o administración, en niveles de trasparencia y respeto de derechos fundamentales, y otra, desde los ciudadanos en los niveles de legitimación de las decisiones; mientras haya mayorías aprobando los destinos y decisiones públicas, mayor será el grado de legitimidad de las mismas y, por ende, los niveles de eficacia.

\section{Desafíos de la Administración Pública frente a una sociedad determinada por las TIC y la globalización}

Como se ha planteado en este ensayo, y a partir de los debates teóricos sobre el problema, la globalización en el campo del derecho manifiesta dos desafíos importantes: uno, relacionado con la crisis de fundamentar el derecho bajo la noción de los Estado-nación, y otro, por la cultura del reconocimiento y respeto de los derechos humanos. Bajo esos dos planteamientos, los Estados de hoy, entre ellos Colombia, han adoptado medidas que le permitan a la Administración Pública insertarse en esa nueva dinámica del derecho, y así responder a los requerimientos de una sociedad moderna o, si se quiere decir, global.

El primer paso significativo dado por el Estado colombiano para que la Administración Pública entrara en la era digital fue la expedición de la Ley 527 de 1999, que reguló todo lo relacionado con el documento electrónico y el comercio electrónico. En su artículo 2. ${ }^{\circ}$ esta ley definió el documento electrónico, conocido también como mensaje de datos, así: "Mensaje de datos. La información generada, enviada, recibida, almacenada o comunicada por medios electrónicos, ópticos o similares, como pudieran ser, entre otros, el Intercambio Electrónico de Datos (EDI), Internet, el correo electrónico, el telegrama, el télex o el telefax". Adicionalmente, en esa ley se dieron las bases para considerar la autenticidad de dichos documentos con la figura de las firmas digitales. Sobre esta indicó:

Firma digital. Se entenderá como un valor numérico que se adhiere a un mensaje de datos y que, utilizando un procedimiento matemático conocido, vinculado a la clave del iniciador y al texto del mensaje, permite determinar que este valor se ha obtenido exclusivamente con la clave del iniciador y que el mensaje inicial no ha sido modificado después de efectuada la transformación.

Esas nociones sirvieron de fundamento para considerar los documentos electrónicos con fines probatorios, y a la vez como vía o canal válido jurídicamente para la interacción del Estado colombiano en sus diferentes servicios (administrativos, judiciales, etc.) con los ciudadanos.

Posteriormente se dieron varios avances, de los cuales resaltamos los siguientes:

- La Ley 1341 de 2009, mediante la cual se definen los principios y conceptos de la sociedad de la información y la organización de las tecnologías de la información y las comunicaciones -TIC-. En esta ley se definen los lineamientos generales para la regulación de las estrategias y políticas públicas en el sector de las tecnologías de la información y las comunicaciones, y además se avanza en el proceso de masificación del gobierno en línea. Sobre 
esto dicha ley estableció en su artículo 2 numeral 8: Con el fin de lograr la prestación de servicios eficientes a los ciudadanos, las entidades públicas deberán adoptar todas las medidas necesarias para garantizar el máximo aprovechamiento de las tecnologías de la información y las comunicaciones en el desarrollo de sus funciones. El Gobierno nacional fijará los mecanismos y condiciones para garantizar el desarrollo de este principio. Y en la reglamentación correspondiente establecerá los plazos, términos y prescripciones, no solamente para la instalación de las infraestructuras indicadas y necesarias, sino también para mantener actualizadas y con la información completa los medios y los instrumentos tecnológicos.

- Posteriormente, la Ley Estatutaria 1712 de 2014, Ley de transparencia y del derecho de acceso a la información pública nacional. Mediante esta Ley se fijaron criterios que hasta antes de la misma habían sido construidos vía jurisprudencial, en relación con los niveles de publicidad o reserva de la información y documentación por parte de las entidades públicas.

De esta Ley se puede resaltar que procura dejar unos límites claros al acceso de la información, y en ese entendido, fijó como criterios orientadores para la interpretación sobre este asunto, la proporcionalidad y la razonabilidad; asimismo, fijó como principios, la transparencia, la buena fe, facilitación, no discriminación, la gratuidad, celeridad, eficacia y calidad de la información. También resulta importante señalar que hizo una serie de definiciones que anteriormente eran problemáticas, como por ejemplo: definió el concepto sobre información pública clasificada como: "Información pública clasificada. Es aquella información que estando en poder o custodia de un sujeto obligado en su calidad de tal, pertenece al ámbito propio, particular y privado o semiprivado de una persona natural o jurídica por lo que su acceso podrá ser negado o exceptuado, siempre que se trate de las circunstancias legítimas y necesarias y los derechos particulares o privados consagrados en el artículo 18 de esta Ley"; también, definió que el concepto de información pública reservada como "aquella información que estando en poder o custodia de un sujeto obligado en su calidad de tal, es exceptuada de acceso a la ciudadanía por daño a intereses públicos y bajo cumplimiento de la totalidad de los requisitos consagrados en el artículo 19 de esta Ley".

Y lo más importante de esta ley fue que obligó a todas las entidades del Estado colombiano y a aquellos particulares que ejercen funciones administrativas, que deberán publicar toda la información pública de su respectiva entidad, mediante los mecanismos digitales, bajo los lineamientos de la estrategia de gobierno en línea. Ello quiere decir que lo que pretendió el legislador es garantizar la transparencia (un elemento de la democratización) mediante la interacción virtual con los administrados.

- En desarrollo de esa ley se expidió el Decreto 103 de 20 de enero de 2015 " por el cual se reglamenta parcialmente la Ley 1712 de 2014 y se articuló con el Decreto 2573 de 2014 más conocido como nuevo decreto de gobierno en línea (GEL). La estrategia de gobierno en línea no es más que una serie de medidas y esquemas de modernización de la actividad estatal en uso de las nuevas tecnologías de la información y comunicación a fin de lograr mayor transparencia en el ejercicio de las actividades del Estado. Esta estrategia se implementó con dicho decreto en las entidades que hacen parte de la rama ejecutiva y, adicionalmente, se señaló que la implementación de la estrategia en las otras ramas del poder público se implementará en virtud de los principios de colaboración y coordinación.

El decreto referenciado establece unos plazos para que la estrategia se cumpla en todas las entidades del Estado. Veamos el esquema que el decreto estableció para las entidades del orden nacional.

Ahora bien, para profundizar este análisis relacionemos los datos mostrados anteriormente, sobre los índices de acceso a las nuevas tecnologías de las comunicaciones, respecto al uso que la ciudadanía les ha dado a todos estos nuevos canales de interacción con 
los ciudadanos, para observar qué tanto las personas han construido una cultura de participar en lo público, es decir, si se ha incentivado la participación democrática a través de las TIC, y si no es así, arriesgarnos a explicar a qué obedecen los patrones de participación democrática presentados.
Las estadísticas que el ministerio de las TIC tiene expuestas al público, sobre la participación ciudadana mediante la estrategia de gobierno en línea muestran los siguientes datos: en un estudio ${ }^{9}$ de percepción de los ciudadanos sobre la estrategia, a escala nacional estos fueron los resultados:

Tabla 1. Sujetos obligados de orden nacional

\begin{tabular}{c|c|c|c|c|c|c}
\hline componentelaño & $\mathbf{2 0 1 5}$ & $\mathbf{2 0 1 6}$ & $\mathbf{2 0 1 7}$ & $\mathbf{2 0 1 8}$ & $\mathbf{2 0 1 9}$ & $\mathbf{2 0 2 0}$ \\
\hline TIC para servicios & $90 \%$ & $100 \%$ & $\begin{array}{c}\text { Mantener } \\
100 \%\end{array}$ & $\begin{array}{c}\text { Mantener } \\
100 \%\end{array}$ & $\begin{array}{c}\text { Mantener } \\
100 \%\end{array}$ & $\begin{array}{c}\text { Mantener } \\
100 \%\end{array}$ \\
\hline $\begin{array}{c}\text { TIC para el Gobierno } \\
\text { Abierto }\end{array}$ & $90 \%$ & $100 \%$ & $\begin{array}{c}\text { Mantener } \\
100 \%\end{array}$ & $\begin{array}{c}\text { Mantener } \\
100 \%\end{array}$ & $\begin{array}{c}\text { Mantener } \\
100 \%\end{array}$ & $\begin{array}{c}\text { Mantener } \\
100 \%\end{array}$ \\
\hline TIC para la Gestión & $25 \%$ & $50 \%$ & $80 \%$ & $100 \%$ & $\begin{array}{c}\text { Mantener } \\
100 \%\end{array}$ & $\begin{array}{c}\text { Mantener } \\
100 \%\end{array}$ \\
\hline $\begin{array}{c}\text { Seguridad y } \\
\text { privacidad de la } \\
\text { Información }\end{array}$ & $40 \%$ & $60 \%$ & $80 \%$ & $100 \%$ & $\begin{array}{c}\text { Mantener } \\
100 \%\end{array}$ & $\begin{array}{c}\text { Mantener } \\
100 \%\end{array}$ \\
\hline
\end{tabular}

Fuente: Imagen tomada del Decreto 2573 de 2014.

Tabla 2. Contrastacion TIC frente a contextos de la comunicación

\begin{tabular}{|c|c|c|c|}
\hline Total país & $\begin{array}{l}\text { ¿Sabe que } \\
\text { puede...? }\end{array}$ & $\begin{array}{l}\text { ¿Sabe } \\
\text { cómo...? }\end{array}$ & $\begin{array}{l}\text { ¿Durante el } \\
\text { año } 2013 \ldots ?\end{array}$ \\
\hline $\begin{array}{l}\text { a) Relacionarse con las entidades públicas para obtener infor- } \\
\text { mación, realizar trámites, obtener servicios, presentar peticio- } \\
\text { nes, quejas o reclamos y participar en la toma de decisiones } \\
\text { haciendo uso de los canales o modelos electrónicos }\end{array}$ & $90,20 \%$ & $79,80 \%$ & $59,50 \%$ \\
\hline b) obtener información de las entidades públicas & $82,80 \%$ & $64,40 \%$ & $41,50 \%$ \\
\hline $\begin{array}{l}\text { c) obtener información sobre los trámites y/o servicios que ofre- } \\
\text { cen las entidades públicas }\end{array}$ & $80,00 \%$ & $60,90 \%$ & $37,50 \%$ \\
\hline $\begin{array}{l}\text { d) contactar a los funcionarios de las entidades públicas para } \\
\text { solicitar información o procedimientos de la entidad pública o } \\
\text { solucionar dudas }\end{array}$ & $67,30 \%$ & $48,30 \%$ & $26,20 \%$ \\
\hline e) Realizar solicitudes, quejas o reclamos & $73,70 \%$ & $54,80 \%$ & $30,10 \%$ \\
\hline f) realizar trámites u obtener servicios ante entidades públicas & $72,90 \%$ & $53,20 \%$ & $31,10 \%$ \\
\hline g) Realizar pagos de trámites y/o servicios & $68,00 \%$ & $49,70 \%$ & $27,30 \%$ \\
\hline $\begin{array}{l}\text { h) Realizar un acompañamiento y revisión permanente sobre la } \\
\text { gestión de las entidades públicas }\end{array}$ & $43,70 \%$ & $28,10 \%$ & $11,90 \%$ \\
\hline $\begin{array}{l}\text { i) Participar en la definición de normas, planes, programas, } \\
\text { proyectos o iniciativas de desarrollo actuales y futuras de las } \\
\text { entidades públicas. }\end{array}$ & $41,50 \%$ & $26,10 \%$ & $11,50 \%$ \\
\hline
\end{tabular}

Fuente: Estudio contratado por el Fondo de Tecnologías de la Información y las Telecomunicaciones y elaborado por Infometrika Ltda. (2013)

9 Estudio de conocimiento y uso de servicios de gobierno electrónico en ciudadanos, empresas y entidades año 2013. Estudio contratado por el Fondo de Tecnologías de la información y las Telecomunicaciones y elaborado por Infométrika Ltda. http://estrategia.gobiernoenlinea.gov.co/623/articles-7913_conocimiento1_2013.pdf 
Una clasificación de género de la participación ciudadana muestra el siguiente cuadro:

Tabla 3. Contrastación TIC frente a contextos de la comunicacion (pespectivas de genero)

\begin{tabular}{l|c|c}
\hline \multirow{2}{*}{ Masculino } & ¿Sabe que puede...? & $91,1 \%$ \\
\cline { 2 - 3 } & ¿Sabe cómo...? & $81,8 \%$ \\
\cline { 2 - 3 } & ¿Durante el año 2013 ha...? & $64,1 \%$ \\
\hline \multirow{3}{*}{ Femenino } & ¿Sabe que puede...? & $92,3 \%$ \\
\cline { 2 - 3 } & ¿Sabe cómo...? & $85,1 \%$ \\
\cline { 2 - 3 } & ¿Durante el año 2013 ha...? & $66,2 \%$ \\
\hline
\end{tabular}

Fuente: Estudio contratado por el Fondo de Tecnologías de la Información y las Telecomunicaciones y elaborado por Infometrika Ltda. (2013)

Al interpretar y relacionar estos resultados estadísticos con los índicies de uso del Internet y de las redes sociales en Colombia, vemos que no existe necesariamente una relación directa entre el aumento progresivo de la virtualización de las personas o el uso de las nuevas tecnologías, y la participación democrática en las actividades del Estado. Más aún, lo que se evidencia es que a pesar de que hay un aumento en el conocimiento o saber que existen nuevos canales de interacción con las entidades estatales, estos tienen un uso muy reducido.

Las causas de ese poco uso o participación democrática por parte de las personas en la función administrativa pueden ser disimiles, pueden ir desde aspectos técnicos relacionados con la implementación de la estrategia de gobierno en línea, como por ejemplo plataformas caídas o estructuras no intuitivas de las páginas web; otras causas pueden ser relacionadas con la capacidad de las sociedades de adaptación al cambio; estas por ejemplo son: desconfianza a la realización de trámites virtualmente, esquemas difíciles de manejo de las herramientas de gobierno en línea, preferencia de la realización de trámites personalmente o con algún contacto directo con funcionarios de las entidades estatales; y otro grupo de causas están relacionada con la falta de interés en la participación de las decisiones y trámites públicos. Ejemplo de estas puede ser cuando muy a pesar de que la sociedad está altamente relacionada con la virtualidad, su dinámica de transformación y demandas sociales circulan a través de Internet, y además de que conoce y sabe que existen herramientas estatales virtuales para participar de las decisiones públicas, no se interesa o no participa de lo público; entonces las preguntas que surgen del presente análisis: ¿Quiénes son esos pocos que sí participan virtualmente de lo público?, ¿y por qué la mayoría no participa? Esos interrogantes son objeto de la investigación que se está desarrollando aún.

Sobre estas causas aún no se han hecho investigaciones que expliquen este fenómeno social y que puedan darnos luces para encontrar el punto adecuado para lograr la democratización de la función pública. Sin embargo, para entender un poco la ineficacia de las dinámicas del proceso de la democratización podrían utilizarse algunos elementos de la construcción teórica que ha desarrollado la economista y socióloga Saskia Sassen; según esta autora, hay una categoría llamada las lógicas de expulsión que opera así: en la dinámica de las ciudades globales, el mercado financiero, en su búsqueda por concretar en activos las ganancias o capitales, crea una serie de mecanismos del mercado que permiten que unos pocos se apropien del capital, y el resto, bajo esas dinámicas de expulsión, quedan fuera del sistema, esto es, no participan del mismo porque no tienen los elementos para hacerlo (Sassen, 1999). Con fundamento en esta lógica, se pueden explicar, en cierta medida, esas causas.

Los elementos teóricos expresados por Sassen expuestos en sus obras más representativas como La ciudad global y Una sociología de la globalización han sido explorados como categorías maestras, útiles para fundamentar el principio de responsabilidad democrática virtual que a priori se ha asumido y que se podría resumir en "soy responsable de los efectos negativos de las medidas administrativas en tanto no participe en el discurso público virtual". El desarrollo de este principio está en construcción teórica a fin de explorar una vía alterna al callejón sin salida planteado tendente a encontrar un punto de equilibro para la ponderación en sus justas proporciones 
de la participación democrática en la función administrativa en la era de las TIC.

\section{Conclusiones}

La referencia al proceso de la globalización como categoría teórica para explicar la dinámica de la democratización de la función administrativa del Estado colombiano en la nueva era de las tecnologías de la información y comunicación se explica en el hecho de que estas dinámicas sociales que inciden directamente en esa relación no se generan en el interior de los Estados, sino que viajan de otras latitudes y que son proceso conducentes a la construcción de las plataformas necesarias para que actores transnacionales puedan llevar a cabo sus políticas y estrategias.

Lo anterior se evidencia en que toda la estrategia de Gobierno en línea desarrollada por el Estado colombiano ha sido fruto de una agenda política de actores transnacional como la OCDE, y así se reconoció en el decreto que la implementó, el cual textualmente dice:

Colombia aceptó las recomendaciones de la Organización para la Cooperación y el Desarrollo Económico (OCDE) en Estrategias de Gobierno Digital, que insta a los gobiernos a adoptar enfoques más estratégicos para un uso de la tecnología que los impulse a ser más abiertos, participativos e innovadores, a través de acciones tales como el diseño de lineamientos para permitir, orientar y fomentar el uso y re-uso de la información pública, aumentar la apertura y la transparencia, incentivar la participación del público en la elaboración de políticas, proporcionar datos oficiales oportunos y confiables, y gestionar los riesgos de uso indebido de datos, así como aumentar la disponibilidad de los datos en formatos abierto" (Considerando número 12 del Decreto 2573 de 2004).

En consonancia con esa dinámica de la sociedad colombiana, el Estado ha procurado crear todos los mecanismos adecuados para hacer frente a las demandas sociales en una nueva era de las comunicaciones.

En ese sentido, pretender la democratización bajo las dos nociones que se explicaron en este texto, la relacionada con la participación ciudadana y la relacionada con la legitimidad del ejercicio de la función administrativa, implica mucho más esfuerzos y actividades que la sola aplicación de una estrategia que genere la plataforma para que el Estado se relacione con los administrados.

\section{Referencias bibliografícas}

- Atienza, M. (2010). Constitucionalismo, globalización y derecho. En CARBONELL, Miguel y García Jaramillo, Leonardo. El canon neoconstitucional. Madrid, España: Editorial Trota.

- Beck, U. (2008). ¿Qué es la globalización? Barcelona, España: Editorial Paidós.

- Bernal, C. (2009). El neoconstitucionalismo y la normatividad del derecho. Bogotá, Colombia: Universidad Externado de Colombia.

- Dahl, R. (2004). La democracia. Traducida por Silvina Floria, Postdata 10, Diciembre/2004, ISSN 1515-209X (11-55).

- De la Reza, Germán A. (2006). Integración económica en América Latina: hacia una comunidad regional en el siglo XXI. México: Editor Universidad Autónoma Metropolitana. Plaza y Valdez Editores

- Daly, Herman; Schütze, Christian; Beck, Ulrich. (1997). Crisis ecológica y sociedad. Valencia, España: Editorial Germania.

- Giddens, A. (2004). Consecuencias de la Modernidad, Madrid, España: Editorial Alianza.

- Hernández Martín, Miguel A. (1988). La intervención del sector público en la economía y su actividad empresarial. Volumen 3 de Acta Salmanticensia Series. Salamanca, España. Editorial Universidad de Salamanca.

- Mojica, J. (2013). El acto administrativo: discurso constitucional en el Estado social de derecho. Bogotá, Colombia. Universidad Nacional de Colombia, Bogotá. Editorial Ibáñez.

- Sassen, S. (1999). La ciudad global. Buenos Aires, Argentina: Editorial Universidad de Buenos Aires.

- Teubner, G. (2000). La fuerza del derecho. Bogotá, Colombia: Siglo del Hombre, Uniandes, Instituto Pensar.

- Twining, W. (2003). Cartografiar el derecho. En Derecho y globalización. Bogotá, Colombia: Siglo del Hombre, Uniandes: Instituto Pensar, 173-207. 